\title{
Inutilizzabilità delle prove e delitto di tortura nel sistema processuale italiano
}

\author{
Evidence's prohibition on use and incrimination of \\ torture in the Italian criminal procedure
}

\author{
Pasquale Bronzo ${ }^{1}$ \\ Università degli Studi di Roma La Sapienza, Italia \\ pasquale.bronzo@uniroma1.it \\ orcid.org/0000-0002-9374-2235
}

Guido Colaiacovo ${ }^{2}$

Università degli Studi di Foggia, Italia

guido.colaiacovo@unifg.it

orcid.org/0000-0001-8618-6632

\begin{abstract}
AвSTRACT: Gli autori esaminano la nuova fattispecie di inutilizzabilità introdotta nel codice di procedura penale italiano contestualmente all'inserimento nel codice penale della previsione che incrimina la tortura. Dopo aver tratteggiato le origini della disciplina e i connotati dell'illecito, affrontano le questioni di carattere processuale per rivolgere l'attenzione su un tema particolarmente complesso e dibattuto che attiene alle conseguenze che l'inutilizzabilità può produrre non soltanto sull'atto compiuto in violazione di un divieto, ma anche sugli altri atti ad esso collegati e, in particolare, sulle prove raccolte nel processo inquinato da condotte di tortura.
\end{abstract}

PAROLE CHIAVE: Reato; Tortura; Prova; Inutilizzabilità.

ABSTRACT: The Authors examine the prohibition on use newly introduced in the criminal procedure code along with the new incrimination of torture's conduct,

1 Professore associato di diritto processuale penale, Università degli Studi di Roma La Sapienza. Dottore in giurisprudenza.

2 Ricercatore di diritto processuale penale, Università degli Studi di Foggia. Dottore in giurisprudenza. Avvocato. 
introduced in the penal code. After having summarized the normative origins and the crime's outlines, Authors deal with its processual issues in order to draw attention about a particularly complex and debated problem: the consequences of the prohibition on use on a deed performed breaking the prohibition, and its consequences on other logically or juridically connected deeds (in particular, on evidences gathered in a trial corrupted by torture acts).

KEYWORDS: offense; torture; evidence; prohibition on use.

SOMmARIO: 1. Premessa. - 2. Alle origini della normativa italiana contro la tortura. - 3. L'incriminazione della tortura. - 4. L'inutilizzabilità di informazioni e dichiarazioni ottenute con la tortura. - 5. Tortura e inutilizzabilità derivata. 6. Considerazioni conclusive.

\section{Premessa.}

La pratica della tortura, ossia l'inflizione o la minaccia di un male nei confronti di una persona per muoverla a collaborare nell'accertamento dei fatti, ha caratterizzato, anche se in tempi ormai remoti, la struttura del rito penale, soprattutto nella tradizione inquisitoria, tanto che processo penale e tortura hanno condiviso lo stesso percorso lungo secoli di storia ${ }^{3}$.

Soltanto lo stimolo del movimento illuminista diede avvio al progressivo abbandono delle regole d'ancien regime e al ripudio di un simile strumento di indagine, tanto violento quanto inaffidabile ${ }^{4}$.

3 Per l'inquadramento della questione sotto il profilo storico è interessante richiamare le ricostruzioni di MANZINI, Vincenzo, Trattato di diritto processuale penale italiano, vol. I. Torino: Utet, 1931, p. 5 e ss. e di CORDERO, Franco Procedura penale, IX ed. Milano: Giuffrè, 2012, p. 24: il confronto tra le due opere permette di notare come la condanna di tale "aberrante istituto" (Manzini, Trattato, cit., p. 5) sia comune, nonostante la siderale distanza che separa la concezione del processo penale propugnata dai due Autori (il primo estensore del Codice Rocco del 1930, espressione giuridica di una visione politica totalitaria dello Stato; l'altro strenuo avversario di tale modello processuale e ispiratore della concezione accusatoria alla base del codice vigente).

4 Chiarissime le parole di BECCARIA, Cesare, Dei delitti e delle pene. Milano: Mondadori, 1991, p. 41 «fortunato sintetizzatore e divulgatore delle dottrine filosofico-sociali del secolo XVIII» (MANZINI, Vincenzo, Trattato, cit., p. 16). 
Fino a quel momento, era ben radicata, e pressochè incontrastata, l'idea che il corpo dell'inquisito fosse materia vile sulla quale incrudelire, dal punto di vista psichico prima ancora che da quello fisico ${ }^{5}$, per vincerne la resistenza. Essendo l'imputato il depositario della verità ${ }^{6}$, la pratica dei tormenti era diffusa nei tribunali, socialmente condivisa nei principi e accreditata dalla scienza criminalistica ${ }^{7}$.

Le origini remote, le suggestioni offerte da questa tematica e l'imponente mole di materiale sedimentato in anni di studi impediscono, in questa sede, anche la più rapida digressione ${ }^{8}$.

Conviene limitarsi a dire, in guisa di premessa, che lo sviluppo di una differente concezione del rapporto tra l'autorità giudiziaria e

5 La tortura, infatti, aveva inizio con una mera pressione psicologica: i giudici inquisitori procedevano con gradualità, cercando di provocare la confessione senza arrivare al tormento vero e proprio: si mostrava anzitutto all'accusato la camera dei supplizi con i suoi vari marchingegni, invitandolo a confessare. Poi, in caso di diniego, lo si denudava e si applicavano ai pollici e alle gambe gli strumenti del tormento - viti, lamelle di ferro, tenaglie senza però infliggere dolore. Solo in caso di perdurante rifiuto a confessare, si procedeva: con l'avvertenza, tuttavia, sempre ripetuta nelle dottrine del tardo Medioevo di limitarsi a reiterare la tortura per non più di tre volte, con dovuti intervalli (cfr. ALESSI, Giorgia, Il processo penale. Profilo storico. Roma - Bari: Laterza, 2001, p. 71).

6 FOSCHINI, Gaetano, Sistema del diritto processuale penale, II ed., vol. I. Milano: Giuffrè, 1965, p. 435.

7 Si pensi che i trattatisti dell'epoca si erano esercitati persino in minuziose ricostruzioni della soglia indiziaria sufficiente per sottoporre l'imputato ai tormenti e sulla descrizione degli stessi (a titolo esemplificativo, si veda la descrizione dei vari gradi della tortura riportata in MANZINI, Vincenzo, Trattato, cit., p. 44, con puntuali richiami ad opere di criminalisti del tempo; sul formalismo della tortura, anche ALESSI, Giorgia, Il processo penale, cit., p. 71).

8 Sul principio per cui l'imputato nel processo penale non può essere obbligato a contribuire all'accertamento dei fatti, cfr., per tutti, GREVI, Vittorio, Nemo tenetur se detegere. Interrogatorio dell'imputato e diritto al silenzio nel processo penale italiano. Milano: Giuffrè, 1972; MAZZA, Oliviero, L'interrogatorio e l'esame dell'imputato nel suo procedimento. Milano: Giuffrè, 2004; LUPARIA, Luca, La confessione dell'imputato nel sistema processuale penale. Milano: Giuffrè, 2006; per ulteriori citazioni e richiami di dottrina e giurisprudenza sul tema dell'imputato nella dimensione probatoria, sia consentito il rinvio a COLAIACOVO, Guido, L'imputato, in SPANGHER, Giorgio; MARANDOLA, Antonella; GARUTI, Giulio; KALB, Luigi (a cura di), Procedura penale. Teoria e pratica del processo. Torino: Utet, 2015, p. 294 e ss. 
l'individuo ha limitato fortemente i poteri della prima sul secondo, tanto che i termini della questione sono ormai orientati dal riconoscimento di diritti e tutele che regolano le intrusioni e gli strumenti di coercizione e impediscono la strumentalizzazione della persona ad eruendam veritatem ${ }^{9}$. Questi principi, che permeano tutto il sistema processuale, coinvolgono necessariamente anche il diritto delle prove, ad evitare che l'esigenza di investigare e raccogliere gli elementi necessari per la repressione del crimine prevarichino oltremodo i diritti di libertà del singolo e, prima tra questi, l'inviolabilità della propria persona. Anche in questo ambito, dunque, la ricerca di un punto di equilibrio impone al legislatore, innanzitutto, di escludere strumenti inutilmente invasivi; poi, di regolare quelli ammessi secondo un criterio di proporzionalità tra il fine perseguito e la compressione degli interessi confliggenti ${ }^{10}$; infine, di apprestare rimedi idonei a reprimere le violazioni delle previsioni che tutelano i diritti fondamentali e a togliere valore processuale al materiale in quel modo raccolto.

Il codice di procedura penale italiano attualmente vigente individua nella sanzione della inutilizzabilità la reazione che presidia il rispetto delle regole probatorie ed espelle le prove raccolte in violazione dei divieti

9 Sulla questione particolare dell'intangibilità fisica del corpo dell'imputato e le relative deroghe consentite per fini investigativi e probatori, ALESCI, Teresa, Il corpo umano fonte di prova. Padova: Cedam, 2017.

${ }^{10}$ Il principio di proporzionalità si va affermando nel panorama giuridico contemporaneo quale cardine delle disposizioni che incidono sui diritti fondamentali (in tema, NEGRI, Daniele, Compressione dei diritti di libertà e principio di proporzionalità, in AA.VV., Diritti della persona e nuove sfide del processo penale. Milano: Giuffrè Francis Lefbvre, 2019, p. 55). In ambito europeo è comparso per la prima volta, come criterio regolatore dell'attività investigativa a seguito del recepimento della direttiva 2014/41/UE sull'ordine europeo di indagine: l'art. 7 del d. lgs. 21 giugno 2017, n. 108 stabilisce, infatti, che l'ordine di indagine non è proporzionato se dalla sua esecuzione può derivare un sacrificio ai diritti dell'imputato, dell'indagato o di altre persone coinvolte dal compimento degli atti richiesti, non giustificato dalle esigenze investigative o probatorie del caso concreto, tenuto conto della gravita' dei reati per i quali si procede e della pena per essi prevista (in tema, SPAGNOLO, Paola, L'ordine europeo di indagine penale, in MARCHETTI, Maria Riccarda; SELVAGGI, Eugenio (a cura di), La nuova cooperazione giudiziaria penale. Padova: Cedam, 2019, p. 288). 
stabiliti dalla legge (art. 191 c.p.p.) ${ }^{11}$. Tali divieti, infatti, tratteggiano le direttrici epistemologiche ed etiche sulle quali si fonda l'opzione cognitiva, escludendo non soltanto gli strumenti privi di attitudine euristica, ma anche quelli che, pur idonei a perseguire un simile scopo, incontrano uno sbarramento innalzato dalla necessità di rispettare i diritti dell'individuo ${ }^{12}$.

Questa impostazione comporta il netto rifiuto della tortura quale strumento investigativo e impone l'allestimento di un apparato normativo teso a reprimere eventuali abusi degli inquirenti con sanzioni particolarmente efficaci e dissuasive, tanto dal punto di vista sostanziale, che dal punto di vista processuale.

\section{Alle origini della normativa italiana contro la tortura.}

L'unanime condanna della tortura è consacrata in trattati e convenzioni che sin dal 1948 si sono susseguiti nel panorama internazionale, imponendo agli Stati aderenti di prevedere espressamente una incriminazione di tale condotta ${ }^{13}$. Non è fuori di luogo ricordare, tuttavia, che, a prescindere dalle sollecitazioni sovranazionali, un chiaro obbligo di penalizzazione era già contemplato dall'art. 13 , comma 4, Cost.,

11 Sul punto, CONTI, Carlotta, L'inutilizzabilità, in MARANDOLA, Antonella, Le invalidità processuali. Profili statici e dinamici. Torino: Utet, 2015, p. 98.

12 In questo senso, CONTI, Carlotta, L'inutilizzabilità, cit., p. 98.

13 A titolo esemplificativo, si possono richiamare la Dichiarazione universale dei diritti dell'Uomo del 1948, lo Statuto di Roma sulla Corte penale internazionale, l'art. 3 della Convenzione europea dei diritti dell'uomo e, soprattutto, la Convenzione contro la tortura e altre pene o trattamenti crudeli, inumani o degradanti approvata dall'Assemblea generale O.N.U. il 19 dicembre 1984, ratificata dall'Italia con 1. 3 novembre 1988, n. 498, e l'art. 3. Quest'ultima, all'art. 4 impone agli Stati aderenti di adeguare il loro diritto penale attraverso l'introduzione di sanzioni adeguate a reprimere gli atti di tortura. Il divieto di tortura, peraltro, ha assunto carattere di norma cogente e la relativa incriminazione rientra tra i crimina iuris gentium di competenza della Corte penale internazionale (NEGRI, Stefania, "Violazioni strutturali" e ritardo nell'esecuzione delle sentenze CEDU: il caso Cestaro c. Italia e l'incerta introduzione del reato di tortura nel codice penale italiano. Diritto penale e processo, Milano, n. 12, p. 1659, 2016). 
in forza del quale, appunto, «è punita ogni violenza fisica e morale sulle persone comunque sottoposte a restrizioni di libertà ${ }^{14}$.

Nella dimensione legislativa il problema appariva risolto soltanto parzialmente: scomparsa da tempo da codici e leggi di diritto processuale quale possibile strumento per la indagini o la ricerca della prova, la tortura non aveva ancora assunto le sembianze del reato ${ }^{15}$.

Eppure, con preoccupante frequenza, le cronache italiane lasciano emergere - talvolta in maniera quasi impercettibile, altre volte in modo più clamoroso - episodi di indagini basate su metodi violenti ${ }^{16} \mathrm{che}$, peraltro, a volte rivelavano una furia cieca tesa solo a produrre sofferenze nella vittima, piuttosto che a facilitare il rilascio di dichiarazioni utili per le indagini ${ }^{17}$.

${ }_{14}$ In questo senso, PELISSERO, Marco, L'introduzione del delitto di tortura nell'ordinamento italiano, in GIARDA, Angelo; GIUNTA, Fausto; VARRASO, Gianluca (a cura di), Dai decreti attuativi della legge "Orlando" alle novelle di fine legislatura. Padova: Cedam, 2018, p. 225, e PEZZIMENTI, Carmela, Tortura e diritto penale simbolico: un binomio indissolubile. Diritto penale e processo, Milano, n. 2, p. 154, 2018.

15 In realtà, come più volte sostenuto dal Governo italiano nel corso delle interlocuzioni con gli organi sovranazionali, condotte di tal fatta ben potevano essere inquadrate in una pluralità di previsioni del codice penale - come, ad esempio, i delitti di abuso d'ufficio (art. 323 c.p.), percosse (art. 581), lesioni personali (art. 582), sequestro di persona (art. 605), arresto illegale (art. 606), indebita limitazione della libertà personale (art. art. 607), abuso di autorità contro arrestati o detenuti (art. 608), perquisizioni e ispezioni personali arbitrarie (art. 609), violenza privata (art. 610) - tutte però accomunate dalla mitezza del trattamento punitivo e dalla blanda capacità dissuasiva, come osservato anche dai giudici dei diritti fondamentali (sul punto, NEGRI, Stefania, "Violazioni strutturali”, cit., p. 1660).

16 Su questo versante, è particolarmente interessante il giudizio di revisione che si è celebrato dinanzi alla Corte d'appello di Perugia che ha assolto il condannato, coinvolto in vicende di terrorismo, dopo aver accertato che la sua confessione era stata estorta con metodi brutali (App. Perugia, 15 ottobre 2013. Disponibile in http://www.archiviopenale.it/tortura--corte-d-app-perugia-26-novembre2013-(ud-15-ottobre-2013)-triaca-con-osservazioni-a-prima-lettura-di-f-loschi/ contenuti/3202).

17 Tra i tanti casi, si può ricordare la vicenda di Stefano Cucchi: arrestato perchè sospettato di aver violato la normativa in materia di stupefacenti, Stefano Cucchi rimaneva vittima di un brutale pestaggio in una caserma dei Carabinieri e decedeva alcuni giorni dopo in un reparto di ospedale nel quale era stato ricoverato. L'iter processuale, che si è concluso a oltre dieci anni di 
Proprio la necessità di adeguarsi finalmente alla Costituzione e agli altri impegni assunti in sede sovranazionale ha imposto all'Italia di intervenire sul codice penale con una novella che ha inserito il delitto di tortura e la relativa istigazione a commetterla. La spinta a colmare la lacuna è venuta da una condanna pronunciata dalla Corte europea dei diritti dell'uomo che ha censurato l'ordinamento italiano, ai margini di una eclatante vicenda giudiziaria, nella quale gli ostacoli alle indagini frapposti dalle forze di polizia e il decorso del tempo avevano comportato, per l'intervenuta prescrizione, l'impunità di autori di gravissimi reati commessi in danno di persone private della libertà personale ${ }^{18}$. La decisione dei giudici dei diritti fondamentali ha ravvisato due carenze: l'inadeguatezza strutturale del sistema discende dal fatto che rende difficoltosa, se non preclude addirittura, la possibilità di sanzionare gli atti di tortura e fa venir meno qualsiasi effetto deterrente ${ }^{19}$.

La 1.14 luglio 2017, n. 110 ha agito, dunque, nella duplice dimensione del diritto penale sostanziale e del diritto processuale: per un verso, ha inserito due nuove incriminazioni, finalizzate a punire la tortura e l'istigazione a commetterla; per altro verso, ha introdotto altre previsioni tese a sterilizzare i contributi investigativi raccolti attraverso metodi violenti. A tali disposizioni, che costituiscono il "cuore" dell'intervento normativo se ne affiancano altre che impongono l'estradizione ed escludono l'immunità per coloro che sono ricercati per crimini di tal fatta ovvero, specularmente, impediscono il respingimento, l'espulsione e l'estradizione di coloro che ne siano vittime. L'intervento normativo - si

distanza dal fatto, ha consentito di accertare che le responsabilità degli autori del reato - che non avevano agito per finalità "investigative", ma per pura brutalità - furono nascoste anche a causa dell'atteggiamento tenuto dai superiori e da manovre tese a sviare le indagini. C. eur. dir. uomo, 7 aprile 2015, Cestaro c. Italia, in Cassazione penale, Milano, n. 10, p. 3796, 2015, e successivamente, C. eur. dir. uomo, 22 giugno 2017, Bartesaghi e altri contro Italia, ivi, n. 10, p. 3773, 2017, entrambe relative agli episodi accaduti in occasione del G8 di Genova del 2001. Sul tema, COLELLA, Angela, C'è un giudice a Strasburgo. In margine alle sentenze sui fatti della Diaz e di Bolzaneto: l'inadeguatezza del quadro normativo italiano in tema di repressione penale della tortura. Rivista italiana di diritto e procedura penale, Milano, n. 4, p. 1801; NEGRI, Stefania, "Violazioni strutturali”, cit., p. 1657. 
è lucidamente osservato - è stato tanto cauto nella repressione penale delle condotte quanto "vigoroso" nel trattamento processuale delle informazioni raccolte attraverso l'uso di queste pratiche, e la nuova previsione sull'inutilizzabilità di informazioni e dichiarazioni estorte con la tortura stimola diverse riflessioni ${ }^{20}$.

La norma interpolata nell'art. 191, comma 2-bis, del codice di rito, infatti, mira a rendere impermeabile il processo rispetto ai risultati informativi provenienti da condotte lesive dei diritti fondamentali. Dunque, se è su questo versante che il legislatore ha introdotto le novità più significative, è opportuno soffermarsi su tale innesto e su eventuali conseguenze di più ampio respiro nel campo del diritto delle prove. Si prospetta, all'orizzonte, un nuovo terreno di scontro sul quale tornano a misurarsi le teorie del male captum, bene retentum, da una parte, e dei "frutti dell'albero avvelenato", dall'altra. La prima afferma che le regole che assegnano valore di prove alle informazioni prodotte nel processo sono tutte contenute nella legge processuale, senza che assuma rilevo la natura illecita della loro raccolta se non è la legge processuale ad assegnare rilievo a tale violazione $^{21}$; la seconda sostiene che determinati vizi che affliggono una prova non possono non incidere sulle prove che da questa "dipendono" 22 .

20 In effetti, se dal punto di vista sostanziale, l'intervento legislativo è stato sottoposto a notevoli critiche, dal punto di vista processuale si sostiene che ben più incisivi sono gli strumenti di tutela della vittima di tortura che non patisce alcuna conseguenza probatoria sfavorevole dalla confessione estorta (CASSIBBA, Fabio, Brevi riflessioni sull'inutilizzabilità delle dichiarazioni estorte con tortura ai sensi del nuovo art. 191, comma 2-bis, c.p.p., Diritto penale contemporaneo, Milano, n. 4, p. 109, 2018).

21 Nel nostro ordinamento manca una disposizione come quella contenuta nel codice di rito brasiliano all'art. 157, che esclude l'utilizzabilità delle prove illecite, intendendo tali le prove assunte in violazione di una norma costituzionale o di legge ordinaria e colpisce con identica sanzione anche le prove che da tali condotte derivino, eccezion fatta qualora non sia evidenziato il nesso tra le une e le altre ovvero quando la prova avrebbe potuto essere acquisita attraverso una diversa e lecita fonte. A tale divieto corrisponde, nella Costituzione, l'art. V, comma LVI, in forza del qualesono inammissibili le prove ottenute con metodi illeciti. Sul tema, ROSSO NELSON, Rocco Antonio Rangel, La prova illecita nell'ordinamento processuale penale brasiliano, https:// archiviodpc.dirittopenaleuomo.org/upload/5017-nelson2018a.pdf.

22 FERRUA, Paolo, Prove illegittimamente acquisite: passato ed avvenire di un'illustre teoria. Diritto penale e processo, Milano, n. 9, p. 1249, 2020. 


\section{L'INCRIMINAZIONE DELLA TORTURA.}

Conviene muovere da un breve inquadramento della previsione incriminatrice, al fine delinearne i tratti caratteristici, utili per l'analisi della materia processuale. L'integrazione della fattispecie di reato costituisce, infatti, il presupposto per l'applicazione della nuova fattispecie di inutilizzabilità ad esso collegata.

L'art. 613-bis c.p., collocato tra i delitti contro la libertà individuale, punisce chiunque con violenze e minacce gravi, ovvero agendo con crudeltà, cagiona acute sofferenze fisiche o un verificabile trauma psichico a una persona privata della libertà personale $\mathrm{o}$ affidata alla sua custodia, potestà, vigilanza, controllo, cura o assistenza ovvero che si trovi in condizioni di minorata difesa. Non conta il fine perseguito: oltre alla tortura "giudiziaria" cadono nella previsione pure tormenti tesi soltanto ad umiliare o annichilire la vittima, benchè la previsione di inutilizzabilità delle informazioni estorte sia destinata ad operare prevalentemente in relazione a condotte del primo tipo. A sottolineare l'intento del legislatore di colpire con efficacia le condotte in discorso è la parallela introduzione di una previsione "complementare" - l'art. 613-ter c.p. - che punisce in modo severo già la mera condotta istigatoria del pubblico ufficiale $o$ dell'incaricato di pubblico servizio ${ }^{23}$.

Un rapido esame della fattispecie consente di affermare che si tratta di una incriminazione dall'ampio spettro operativo che - si può dire in una prospettiva di sintesi - mira a stigmatizzare le condotte di violenza, fisica o morale, compiute da una persona che riveste una posizione di preminenza, nei confronti di un'altra che si trova rispetto all'agente in una posizione di soggezione ${ }^{24}$. Deve trattarsi, comunque, di condotte che, pure

23 Per l'inquadramento della previsione, PELISSERO, Marco, L'introduzione del delitto di tortura, cit., p. 225 e PEZZIMENTI, Carmela, Tortura e diritto penale simbolico, cit., p. 155 e ss.

24 Le prime applicazioni pratiche confermano questa tendenza e, peraltro, confermano che, a prescindere dalla occasio legis, non sono avvenute per sanzionare in via esclusiva condotte delittuose di componenti delle forze dell'ordine. Ad esempio, nel caso di Cass., sez. V, 8 luglio 2019, n. 47079, in Cassazione penale, Milano, n. 6, p. 2340, 2020, con osservazioni di CAROLEO GRIMALDI, Nunzio, alcuni minorenni avevano reiteratamente compiuto incursioni notturne in danno di una persona affetta da disturbi psichiatrici. 
isolate, si rivelino particolarmente crudeli: l'intensità dell'aggressione è, infatti, il tratto che distingue questo reato da altre figure simili.

Ancorchè ispirata dall'intenzione di delimitare con precisione il campo applicativo dell'incriminazione, per evitare che possa essere d'ostacolo allo svolgimento delle ordinarie attività di indagine penale, la trama della disposizione rischia però di complicare il lavoro dell'interprete, se è vero quanto si è detto a proposito di un eccesso nella individuazione delle modalità d'azione ${ }^{25}$.

Dunque, la fattispecie penale è costruita come reato comune, che può essere compiuto da chiunque, e richiede, sotto il profilo soggettivo, il dolo generico ${ }^{26}$. Qui, l'Italia si è discostata dalle disposizioni sovranazionali che, invece, richiedono sia il dolo intenzionale, sia il dolo specifico ${ }^{27} \mathrm{e}$ mettono al bando soltanto la cosiddetta "tortura di Stato" ossia la tortura diretta ad acquisire gli elementi necessari per le indagini penali ${ }^{28}$.

25 In questo senso, PELISSERO, Marco, L'introduzione del delitto di tortura, cit., p. 233, e PEZZIMENTI, Carmela, Tortura e diritto penale simbolico, cit., p. 156. È un difetto che riverbera conseguenze negative anche sul versante processuale: come si vedrà, infatti, la puntuale individuazione delle condotte penalmente rilevanti è fondamentale anche per delineare il campo applicativo della sanzione di inutilizzabilità degli atti raccolti attraverso la tortura.

In realtà, come ricorda PELISSERO, Marco, L'introduzione del delitto di tortura, cit., p. 229, nel corso dei lavori preparatori la maggior parte dei disegni di legge prevedeva l'introduzione di un reato proprio, sulla falsariga, quindi, delle previsioni sovranazionali. Per un'ampia e puntuale ricostruzione dell' $i$ ter legislativo, COLELLA, Angela, La repressione penale della tortura: riflessioni de iure condendo. Disponibile in https://archiviodpc.dirittopenaleuomo. org/upload/1406048334COLELLA_2014a.pdf

In questo senso è chiarissimo ancora l'art. 1 della Convenzione del 1984 (sul punto, PELISSERO, Marco, L'introduzione del delitto di tortura, cit., p. 242).

Ad esempio, secondo l'art. 1 della Convenzione del 1984, la tortura indica qualsiasi atto mediante il quale sono intenzionalmente inflitti ad una persona dolore o sofferenze forti, fisiche o mentali, al fine segnatamente di ottenere da essa o da una terza persona informazioni o confessioni, di punirla per un atto che essa o una terza persona ha commesso, o è sospettata aver commesso, di intimorirla o di far pressione su di lei o di intimorire o di far pressione su una terza persona, o per qualsiasi altro motivo fondato su qualsiasi forma di discriminazione, qualora tale dolore o sofferenze siano inflitte da un agente della funzione pubblica o da ogni altra persona che agisca a titolo ufficiale, o su sua istigazione, o con il suo consenso espresso o tacito. Sulla scelta del legislatore di non perseguire soltanto la "tortura di Stato", Cass., sez. V, 8 luglio 2019, n. 47079, cit. 
Quest'ultima, comunque, è oggetto dello specifico trattamento penale descritto dal secondo comma, che inasprisce la pena qualora il fatto sia compiuto da un pubblico ufficiale o da un incaricato di un pubblico servizio, con abuso dei poteri o in violazione dei doveri inerenti alla funzione o al servizio ${ }^{29}$. In questa declinazione, infatti, la norma consente di reprimere soprattutto aggressioni compiute in occasione di attività di polizia e si collega direttamente con gli eventi dai quali è scaturita la novella.

Numerose, dunque, sono le situazioni contemplate dal legislatore, ma le più significative, dall'angolo visuale del processualista, sono quelle relative al compito di custodia, vigilanza o controllo affidato al soggetto agente e alla privazione della libertà personale della vittima. È immediato, infatti, il collegamento con la coercizione processuale - precautelare o cautelare - ed esecutiva e con la correlata necessità di garantire una specifica e ulteriore protezione -- in rima con l'art. 13 Cost. - per coloro che siano privati della libertà personale. Poichè, tuttavia, non può escludersi che la cattura dell'imputato o del condannato e l'esigenza di evitare la sua fuga richiedano, oltre la privazione della libertà, anche metodi energici, a volte imposti dalla necessità di vincere eventuali resistenze, il legislatore ha introdotto una peculiare precisazione, contenuta nel terzo comma, che esclude l'applicazione della norma qualora le sofferenze derivino unicamente dall'esecuzione di legittime misure privative o limitative di diritti.

\section{L'INUTILIZZABILITÀ DI INFORMAZIONI E DICHIARAZIONI OTTENUTE ATTRAVERSO LA TORTURA.}

La ricaduta processuale del compimento di condotte di tortura nel corso delle investigazioni è l'inutilizzabilità del materiale raccolto dagli inquirenti.

29 Secondo PELISSERO, Marco, L'introduzione del delitto di tortura, cit., p. 242, non dovrebbe trattarsi di una circostanza aggravante, ma di una fattispecie autonoma: la soluzione risolverebbe una pluralità di questioni interpretative e consentirebbe di dare maggiore significato al disvalore insito nelle condotte di prevaricazione provenienti da esponenti ella pubblica autorità. In questa ottica, ad esempio, consentirebbe di superare i problemi determinati dal bilanciamento con eventuali circostanze attenuanti, che rischierebbe di obliterare il particolare disvalore legato alla qualifica soggettiva dell'autore (sul tema, PEZZIMENTI, Carmela, Tortura e diritto penale simbolico, cit., p. 155). 
La inidoneità probatoria delle dichiarazioni raccolte in questo modo sarebbe desumibile già da altre previsioni: dall'art. 188 c.p.p. che tutela la «libertà morale della persona nella assunzione della prova», in generale, e dall'art. 64, comma 2, c.p.p., per quanto concerne, in particolare, l'imputato ${ }^{30}$. Anzi, mentre alla stregua di queste disposizioni l'inutilizzabilità delle informazioni ex art. 191 c.p.p. scaturisce dal semplice uso di mezzi diretti all'effrazione psichica, l'inutilizzabilità prevista dal nuovo comma 2-bis presuppone la ricorrenza di tutti gli elementi che compongono la correlata fattispecie di reato (le "sofferenze fisiche" o il "verificabile trauma psichico", la molteplicità delle condotte, la violazione della dignità). Ne consegue che la previsione sanziona una sottofattispecie di lesione della libertà morale della persona più articolata e dunque meno agevole da accertare ${ }^{31}$.

Al di là dell'indubbio valore simbolico della novella, quale sottolineatura della centralità della dignità della persona nel processo ${ }^{32}$, essa in primo luogo positivizza l'assunto - in verità ben consolidato sia in dottrina che in giurisprudenza - secondo cui qualsiasi dichiarazione estorta è inutilizzabile nel processo, ma soprattutto, - nell'affermare che

30 L'art. 188 c.p.p. afferma che «non possono essere utilizzati, neppure con il consenso della persona interessata, metodi o tecniche idonei a influire sulla libertà di autodeterminazione o ad alterare la capacità di ricordare e di valutare i fatti», mentre in tema di interrogatorio dell'imputato l'art. 64 comma 2 c.p.p. specifica che «non possono essere utilizzati, neppure con il consenso della persona interrogata, metodi o tecniche idonei a influire sulla libertà di autodeterminazione $\mathrm{o}$ ad alterare la capacità di ricordare e di valutare i fatti». Del resto, già sotto la vigenza del previgente codice del 1930 la dottrina, pur in assenza di previsioni normative espresse, tendeva a estromettere dal processo contributi probatori estorti con metodi simili a quelli che oggi sono contemplati dall'art. 613-bis c.p. Ad esempio, secondo CORDERO, Franco, Procedura penale, cit., p. 619, «nemmeno sotto il vecchio codice costituivano prova le emissioni verbali eterodeterminate, ma era arguibile dal sistema, mancando previsioni ad hoc».

31 GREVI, Vittorio; ILLUMINATI, Giulio, Prove, in CONSO, Giovanni; GREVI, Vittorio; BARGIS, Marta (a cura di), Compendio di procedura penale, IX ed. Padova: Cedam, 2018, p. 319, secondo i quali la precisazione potrebbe rivelarsi addirittura controproducente, prestandosi ad essere erroneamente intesa nel senso che la libertà di autodeterminazione risulti pregiudicata solo in presenza di comportamenti particolarmente gravi e reiterati come quelli descritti dal codice penale.

32 CASSIBBA, Fabio, Brevi riflessioni sull'inutilizzabilità, cit., p. 114. 
dichiarazioni e informazioni ottenute mediante il delitto di tortura non sono «comunque» utilizzabili - vuole chiarire che l'inefficacia probatoria delle stesse si proietta anche al di là del loro impiego nella sentenza.

Quanto alla natura della patologia che colpisce dichiarazioni e informazioni acquisite tramite tortura, si tratta, innanzitutto, di una inutilizzabilità riconducibile nella categoria delle inutilizzabilità "patologiche", o inutilizzabilità-sanzione, che derivano dalla violazione di uno specifico divieto probatorio. Il vizio, pertanto, non potrà essere sanato, ma, anzi, dovrà essere rilevato, anche d'ufficio, in ogni stato e grado del procedimento, a prescindere dalla scelta di definire il rito nelle forme del rito abbreviato o del patteggiamento e da eventuali accordi tra le parti sulla acquisizione di atti al fascicolo per il dibattimento ${ }^{33}$. L'inutilizzabilità opererà poi in qualsiasi altro tipo di giudizio non soltanto penale, ma anche di diversa natura, come ad esempio nel procedimento di prevenzione ${ }^{34}$.

L'unica deroga prevista alla radicale irrilevanza processuale del prodotto di queste condotte è rappresentata dal loro impiego nel processo a carico dei torturatori qualora le dichiarazioni o le informazioni debbano essere spese contro coloro che hanno commesso il delitto e al solo fine di provarne la responsabilità penale ${ }^{35}$. La previsione è peraltro superflua, oltre che di imprecisa formulazione, perché qualsiasi atto probatorio inutilizzabile nel suo contenuto informativo, non può non valere logicamente a provare che l'atto stesso è stato compiuto, in questo caso come corpo del reato ${ }^{36}$.

33 Sul rilievo delle invalidità processuali negli accordi per l'acquisizione di atti, BRONZO, Pasquale, Accordi sul fascicolo e vizi degli atti. Cassazione penale, Milano, n. 11, p. 3764, 2020.

34 Tanto in ossequio all'insegnamento di Sez. un., 25 marzo 2010, n. 13426, in Cassazione penale, 2010, p. 3049, con nota di BELTRANI, Sergio, Intercettazioni inutilizzabili e procedimento di prevenzione: la fine di un equivoco.

35 UBERTIS, Giulio, Sistema di procedura penale, vol. II. Milano: Giuffrè, 2020, p. 228; così anche GREVI, Vittorio; ILLUMINATI, Giulio, Prove, cit., p. 319.

36 C. eur. dir. uomo, 30 giugno 2008, Gäfgen c. Germania [GC], ricorso n. 22978/05, in hudoc.echr.coe.int: per un commento, MAFFEI Stefano, Il mantello della legge. Male captum, bene retentum e dottrina del «ritrovamento inevitabile» in una recente pronuncia della Corte Europea dei diritti dell'uomo, in Studi in onore di Mario Pisani. Milano: Giuffrè, 2010, vol. 2, p. 349 ss. 
L'operatività della previsione, proprio per il suo peculiare significato è collegata all'accertamento della commissione di una condotta rilevante ai sensi dell'art. 613-bis c.p. E non potrebbe essere diversamente alla luce della scelta legislativa di fare esplicito riferimento a quanto ottenuto "mediante il delitto di tortura" ${ }^{37}$. Tuttavia, questo non significa che condotte lesive della libertà morale dell'indagato o di una persona sentita nel corso di una indagine penale che non integrino la fattispecie delittuosa in discorso non siano parimenti 'inutilizzabili' ai sensi delle altre disposizioni normative prima ricordate ${ }^{38}$. Il rapporto tra queste previsioni allora può essere descritto come quello tra un'area più ampia, perimetrata dagli artt. 188 e 64 c.p.p., che al suo interno ne contiene una più ristretta, delimitata dall'art. 191, comma 2-bis c.p.p.

Viene da chiedersi, in primo luogo, quale tipo di valutazione il giudice sia chiamato a compiere e di quali strumenti egli disponga per accertare il compimento della tortura e, in secondo luogo, quali rimedi abbia la persona condannata all'esito di un processo in seno al quale è stata utilizzata la tortura qualora tale situazione divenga nota dopo il passaggio in giudicato della sentenza.

Nulla quaestio nell'ipotesi in cui il reato sia stato accertato con sentenza passata in giudicato: qui il precedente consente facilmente la diagnosi di inutilizzabilità della dichiarazione estorta e, dunque, l'espulsione dal compendio probatorio del materiale inquinato.

Più complicata, invece, è la situazione che si verifica in assenza di un accertamento definitivo del reato. Si tratta, peraltro, di ipotesi destinate ad essere piuttosto frequenti nella pratica: l'accertamento richiede comunque la celebrazione di un processo ad hoc, che correrebbe parallelo a quello in seno al quale le informazioni o le dichiarazioni dovrebbero essere utilizzate.

D'altro canto, la stessa commissione del delitto di tortura potrebbe divenire nota o comunque essere denunciata a distanza di molto tempo:

37 Contra, tuttavia, CASSIBBA, Fabio, Brevi riflessioni sull'inutilizzabilità, cit., p. 113.

38 Ad esempio l'uso di tecniche di ipnosi, senza alcuna coercizione della persona, anzi col suo consenso o su sua richiesta, rientreranno nel campo di applicazione dell'art. 188 c.p.p. 
si tratta di reati che maturano in contesti poco penetrabili dall'esterno, e non è difficile che in quel momento il procedimento pregiudicato dalla condotta illecita si sia ormai concluso.

Nella prima ipotesi - ossia quando la sanzione di inutilizzabilità debba operare rispetto all'impiego delle dichiarazioni ottenute attraverso condotte di tortura in un procedimento penale non ancora concluso - il giudice può e deve compiere ogni accertamento necessario per verificare in completa autonomia il compimento di comportamenti simili in danno dell'imputato o di testimoni. A tal fine, l'indagine potrebbe svolgersi, sulla falsariga di quanto dispone l'art. 500, comma 5, c.p.p, per l'accertamento di condotte illecite volte ad inquinare prove dichiarative, nel contesto di un procedimento incidentale ${ }^{39}:$ l'oggetto di prova in questo caso sarebbe costituito da fatti materiali rilevanti ai fini dell'applicazione di una norma processuale, ai sensi dell'art. 187, comma 2, c.p.p.

Nella seconda ipotesi, invece, l'uso della tortura emerge quando ormai la decisione basata sulle informazioni illecitamente ottenute è già passata in giudicato e, pertanto, occorre rivolgere lo sguardo verso le impugnazioni straordinarie e, precisamente, alla revisione. Anche qui, tuttavia, la soluzione è agevole soltanto in presenza di una sentenza di condanna definitiva a carico dei torturatori, che consentirebbe di esperire il rimedio ai sensi dell'art. 630, lett. d), c.p.p. In tal caso, alla corte d'appello spetta verificare in che modo la commissione del reato abbia inciso sulla statuizione impugnata, individuando nell'economia complessiva delle valutazioni compiute sull'intero compendio probatorio quale sia stato il rilievo delle prove acquisite attraverso la tortura.

39 Si tratta di una ipotesi eccezionale di recupero probatorio di dichiarazioni reste nelle indagini preliminari da persone che poi vengono sentite come testimoni in dibattimento: ai sensi del quarto comma dell'art. 500 c.p.p. «Quando, anche per le circostanze emerse nel dibattimento, vi sono elementi concreti per ritenere che il testimone è stato sottoposto a violenza, minaccia, offerta o promessa di denaro o di altra utilità, affinchè non deponga ovvero deponga il falso, le dichiarazioni contenute nel fascicolo del pubblico ministero precedentemente rese dal testimone sono acquisite al fascicolo del dibattimento e quelle previste dal comma 3 possono essere utilizzate». Il successivo comma 5 precisa che «sull'acquisizione di cui al comma 4 il giudice decide senza ritardo, svolgendo gli accertamenti che ritiene necessari, su richiesta della parte, che può fornire gli elementi concreti per ritenere che il testimone è stato sottoposto a violenza, minaccia, offerta o promessa di denaro o di altra utilità». 
Qualora, invece, una simile sentenza manchi, l'accesso al giudizio di revisione diviene più complicato: la giurisprudenza interpreta l'art. 630 comma 1 lett. d) c.p.p. - che ammette la revisione «se è dimostrato che la condanna venne pronunciata in conseguenza di falsità in atti o in giudizio o di un altro fatto previsto dalla legge come reato»- nel senso che il rimedio sia attivabile solo in presenza una sentenza di condanna che accerti la commissione del reato che ha determinato la decisione da rimuovere, salvo che in quel procedimento sia intervenuta una causa estintiva che abbia impedito un tale accertamento nel merito ${ }^{40}$.

E tuttavia, una impostazione simile - nella quale l'ingiustizia della condanna si somma alla impunità degli autori del reato - lascerebbe senza rimedio statuizioni che poggiano sulla violazione di diritti fondamentali: occorre quindi saggiare ogni possibilità interpretativa per giungere comunque alla rimozione del giudicato.

In questa prospettiva, la lett. c) dell'art. 630 c.p.p., che consente la revisione in presenza di una "prova nuova", può essere individuata quale punto di partenza. In questa ottica, la commissione del reato di tortura costituisce il quid novi da provare poichè la dimostrazione che le investigazioni sono state inquinate da una simile condotta aprirebbe la strada all'applicazione dell'art. 191, comma 2-bis, c.p.p. e alla conseguente eliminazione dal compendio probatorio degli elementi acquisiti mediante tortura. L'accertamento del reato costituisce, come detto poc'anzi, un fatto rilevante per l'applicazione di una norma processuale. Nella fase rescissoria del giudizio di revisione, il giudice dovrebbe nuovamente esprimersi, alla luce di questa nuova conformazione del fascicolo del dibattimento, sulla penale responsabilità dell'imputato.

In ogni caso, a fronte dell'accertata commissione del delitto di tortura, si pone il problema di verificare in concreto quali siano le conseguenze che discendono dall'espunzione del materiale acquisito mediante il suddetto reato dal compendio probatorio. Si ritiene che l'eliminazione di tali elementi imponga comunque di valutare sulla base di ciò che residua la sussistenza della penale responsabilità dell'imputato non potendo estendersi l'effetto invalidante fino a escludere in radice la

40 Cass., sez. V, 24 giugno 2009, n. 40169, in Cassazione penale, Milano, n. 2, p. 632, 2012. 
possibilità di emettere una sentenza di condanna se la stessa possa reggersi su prove immuni dall'effetto inquinante della tortura. Sviluppo coerente di tale impostazione nei giudizi di impugnazione è la conseguenza che la censura incentrata sulla violazione dell'art. 613-bis c.p. deve - nella prospettiva di quella valutazione controfattuale nota come "prova di resistenza" ${ }^{41}$ - delineare la capacità dimostrativa dell'elemento da espellere e la sua incidenza ai fini della decisione ${ }^{42}$.

Resta da chiarire, infine, la sorte di eventuali contributi probatori favorevoli all'imputato. Da questo punto di vista, la questione appare riconducile al dilemma in materia di intercettazioni inutilizzabili e alla possibilità di superare i vizi della procedura e la conseguente inutilizzabilità del materiale captato qualora le conversazioni contengano elementi favorevoli all'imputato. Da tempo in dottrina si pone il quesito se l'inutilizzabilità probatoria operi incondizionatamente, e sia dunque configurabile in ogni caso il divieto di valutazione di qualsiasi prova acquisita contra legem, oppure se possa configurarsi una deroga per l'ipotesi in cui i dati probatori risultino favorevoli alla difesa. Nonostante né il testo dell'art. 191 c.p.p. né quello delle previsioni speciali di inutilizzabilità sparse nel nostro codice di rito offrano argomenti utili in tal senso, qualcuno ammette - per ragioni etiche più che giuridiche ${ }^{43}-$ la

41 Secondo la Suprema Corte, qualora si lamenti l'inutilizzabilità o la nullità di una prova dalla quale siano stati desunti elementi a carico, il motivo di impugnazione deve illustrare, a pena di inammissibilità per aspecificità, l'incidenza dell'eventuale eliminazione del predetto elemento ai fini della cosiddetta "prova di resistenza". Ciò in quanto è sempre necessario valutare se le residue risultanze, nonostante l'espunzione di quella inutilizzabile, risultino sufficienti a giustificare l'identico convincimento. Secondo tale impostazione, gli elementi di prova acquisiti illegittimamente diventano irrilevanti ed ininfluenti se, nonostante la loro espunzione, le residue risultanze risultino sufficienti a giustificare l'identico convincimento (ex plurimis, Cass., sez. fer., 6 agosto 2019, n. 44878, in Guida al diritto, Milano, n. 48, p. 101, 2019). Sul punto, anche infra, § 5.

42 In senso contrario, ROMANELLI, Bartolomeo, Delitto di tortura e inutilizzabilità probatoria, in GIARDA, Angelo; GIUNTA, Fausto; VARRASO, Gianluca (a cura di), Dai decreti attuativi, cit., p. 263, secondo il quale una diversa conclusione sarebbe coerente anche gli arresti dei giudici dei diritti fondamentali, e CASSIBBA, Fabio, Brevi riflessioni sull'inutilizzabilità, cit., p. 115. Sul punto, infra, §5.

43 CORDERO, Franco, Il procedimento probatorio, in Tre studi sulle prove penali. Milano: Giuffrè, 1963, p. 143. 
possibilità di impiego pro reo, ad esempio per le ipotesi in cui la sanzione presidi interessi extraprocessuali, e la nega recisamente solo quando invece è posta a tutela della affidabilità gnoseologica dei dati acquisiti al processo $^{44}$. Nel nostro caso una utilizzabilità pro reo appare decisamente opinabile, anche alla luce dal fatto che informazioni e dichiarazioni estorte con la violenza e la minaccia da persone fisicamente e psicologicamente prostrate presentano l'ulteriore problematica relativa alla loro scarsa attendibilità ${ }^{45}$.

Siccome poi, come detto, la tortura è stata costruita come reato comune - mentre la "tortura di Stato" è punita come una autonoma fattispecie - è possibile che la condotta in discorso sia tenuta anche da soggetti diversi da pubblici ufficiali che agiscono "nell'interesse" di altre parti processuali. In altri termini, una situazione simile si verificherebbe qualora le dichiarazioni fossero estorte su mandato dell'imputato al fine di precostituirsi prove in suo favore. Anche qui, dunque, ove fosse accertata la commissione del reato, il materiale raccolto sarebbe colpito dalla previsione di inutilizzabilità ${ }^{46}$.

44 GALANTINI, Novella, L'inutilizzabilità della prova nel processo penale, Padova: Cedam, 1992, p. 77. In altre ricostruzioni, si ipotizza una utilizzabilità in favorem (solo) per in presenza di una sanzione riguardante il quomodo acquisitivo in quanto in tal caso il risultati probatorio potrebbe essere ottenuto attraverso una condotta rispettosa del dato normativo (LOZZI, Gilberto, Lezioni di procedura penale. Torino: Giappichelli, 2018, p. 201). La dottrina maggioritaria esclude, tuttavia, una limitazione generale (ossia in assenza di limitazioni specifiche) della operatività della sanzione in relazione alla direzione di impiego (contra o in favorem).

45 Ritiene precluso l'utilizzo pro reo anche ROMANELLI, Bartolomeo, Delitto di tortura e inutilizzabilità probatoria cit., p. 264, secondo il quale una diversa soluzione potrebbe incentivare il ricorso alla tortura al fine di precostituire prove liberatorie e proprio o altrui beneficio.

46 A titolo esemplificativo, si può segnalare che la Corte europea dei diritti dell'uomo ha censurato l'uso probatorio della registrazione di dichiarazioni di un soggetto rese mentre veniva sottoposto a tortura dagli altri membri dell'organizzazione criminale, puntualizzando che il divieto di utilizzo delle dichiarazioni sotto tortura per non minare l'equità complessiva del processo non deve essere limitato ai casi di violenza commessi da un pubblico ufficiale, ma pure da un terso privato cittadino (C. eur. dir. uomo, 5 novembre 2020, Cwick c. Polonia). 


\section{TORTURA E INUTILIZZABILITÀ DERIVATA}

Dicevamo che l'art. 191, comma 2-bis, c.p.p., nel ribadire l'inutilizzabilità probatoria delle dichiarazioni e informazioni ottenute attraverso condotte di tortura, già desumibile dal combinato disposto degli artt. 188, 64 comma 2 e 191 comma 1, c.p.p. vale soprattutto a sottolineare - nell'avverbio «comunque» - che l'irrilevanza va oltre il loro impiego diretto in sentenza, essendo esse inidonee a "fare prova" in qualsiasi contesto procedimentale ${ }^{47}$. Facile comprendere i motivi di tale sottolineatura: le dichiarazioni estorte con la tortura quasi mai affiorano alla luce del giudizio dibattimentale e solitamente restano nascoste tra le carte del fascicolo del pubblico ministero, incidendo sulle sorti del processo attraverso la loro influenza sull'orientamento delle indagini e sulla raccolta del materiale probatorio piuttosto che attraverso la loro diretta valutazione nella decisione finale.

Si tratta di un profilo importante, anche per la valenza preventiva della sanzione di inutilizzabilità, alla cui ratio non è estraneo un effetto dissuasivo, ossia di prevenzione delle violazione della regole, oltre che di rimedio alle violazioni già consumate. Le sanzioni processuali rappresentano un fondamentale deterrente per i soggetti che curano la provvista probatoria dall'agire contra legem: chi trasgredisce alla regola non può sperare di avvantaggiarsi del risultato ottenuto ${ }^{48}$.

La clausola va tuttavia intesa giudiziosamente, alla luce dei principi del nostro sistema processuale. Il fatto che la dichiarazione estorta sia "comunque" inutilizzabile implica che la stessa, oltre a non poter essere spesa in sentenza, non possa essere utilizzata neppure in modo "indiretto", e cioè posta a fondamento di atti istruttori che richiedano per il loro legittimo compimento una particolare giustificazione: una intercettazione, una perquisizione, un sequestro di corrispondenza, un provvedimento

47 Anzi, come si è già ricordato, ogni dichiarazione estorta o resa in stato di costrizione è giuridicamente inesistente come prova, poiché la libertà del volere è elemento essenziale e costitutivo di ogni dichiarazione resa nel processo, come osservava Cordero già sotto l'impero del vecchio codice ed in assenza di qualsiasi comminatoria di invalidità (supra, nt. 30).

48 CASSIBBA, Fabio, Brevi riflessioni sull'inutilizzabilità, cit., p. 166; in argomento, volendo BRONZO, Pasquale, Il fascicolo per il dibattimento. Poteri delle parti e ruolo del giudice. Milano: Cedam, 2017, p. 270. 
ammissivo di incidente probatorio. Tanto vale anche per atti di natura non istruttoria, ovviamente: neppure misure cautelari o proroghe delle indagini possono validamente basarsi su informazioni processualmente inutilizzabili. Sintetizzando, l'inidoneità probatoria che discende dalla sanzione riguarda, oltre alla sentenza, ogni altro contesto "decisorio" all'interno del procedimento ${ }^{49}$.

L'avverbio "comunque" e il riferimento alle "informazioni" oltre che alle "dichiarazioni" evoca un effetto riflesso della sanzione processuale molto simile a quello che il nostro codice stabilisce in tema di notizie coperte da segreto di Stato, laddove prevede che l'opposizione del medesimo, «confermata con atto motivato dal Presidente del Consiglio dei Ministri, inibisce all'autorità giudiziaria l'acquisizione e l'utilizzazione, anche indiretta, delle notizie coperte dal segreto» (art. 202, comma 5, c.p.p.).

Non ci sarebbe neppure bisogno di precisarlo, a rigore, accedendo alla ricostruzione propugnata da tempo dalla dottrina più accreditata, secondo la quale le norme sulla "prova" compendiate nel libro terzo del codice di rito non valgono solo per la fase del giudizio, ma devono valere anche per le indagini preliminari ${ }^{50}$. Letto in questo modo, il comma 2-bis dell'art. 191 c.p.p. sarebbe una norma pedagogica, di chiarificazione, utile nella misura in cui sottolinea come in casi del genere i vizi probatori siano in grado di inquinare il processo ben prima che esso giunga alla sentenza.

Con una avvertenza, però: non è che il "vizio" che affligge la dichiarazione estorta si propaghi agli atti istruttori successivi e da essa

49 «La sentenza che decida sulla base di una prova nulla, inutilizzabile o materialmente inesistente non è affetta da alcun tipo di invalidità derivata; è semplicemente viziata nella motivazione, in quanto, omessa quella prova, la motivazione collassa» (FERRUA, Paolo, Prove illegittimamente acquisite, cit., p. 1255).

50 GREVI, Vittorio - ILLUMINATI, Giulio, Prove, cit., p. 305. Quando il legislatore, nella disposizione che prevede che le informazioni provenienti dai confidenti della polizia rimasti anonimi non possono essere «acquisite né utilizzate», ha sentito il bisogno di precisare che «l'inutilizzabilità opera anche nella fasi diverse dal dibattimento» (art. 203 comma 1-bis c.p.p.) reagendo ad una giurisprudenza piuttosto lassista, ha esplicitato una conclusione che era già desumibile dal sistema (in tema, volendo, BRONZO, Pasquale, Le modificazioni in tema di informazioni confidenziali, in LATTANZI, Giorgio (a cura di) Guida al giusto processo. Milano: Giuffrè, 2002, p. 177 ss.). 
dipendenti; accade, piuttosto, che le informazioni frutto di tortura non sono in grado di giustificare il compimento dell'atto istruttorio che richieda una particolare motivazione, come una perquisizione, che ove fondata su tali atti risulterebbe affetta da nullità ex art. 125 comma 3 c.p.p. Una invalidità originaria, dunque.

Il divieto neointrodotto è stato interpretato nel senso che la tortura renda inutilizzabili le dichiarazioni estorte «non solo in vista della prova della responsabilità penale $\mathrm{o}$, comunque, della veridicità dell'affermazione del fatto oggetto delle dichiarazioni, ma anche come spunto investigativo per la ricerca di altri elementi probatori. In breve, la violazione del divieto di impiegare la tortura a fini confessori o di raccolta di dichiarazioni fa scattare l'inutilizzabilità derivata di ogni elemento di prova reperito a partire dalle informazioni estorte ${ }^{51}$.

In realtà, non sembra che il legislatore del 2017 abbia creato un'ipotesi di propagazione di inutilizzabilità delle prove ottenute tramite la tortura (come pure avrebbe potuto fare). Si può qui solo accennare al pluriennale dibattito in ordine alla figura della "inutilizzabilità derivata": una parte della dottrina ritiene applicabile alla inutilizzabilità un meccanismo di diffusione, simile a quello che l'art. 185 c.p.p. prevede per le nullità, in virtù del quale il vizio di una prova si trasmette a prove successivamente raccolte ove tra le due sussista un nesso di dipendenza ${ }^{52}$; secondo una diversa impostazione, invece, nessuna invalidità derivata potrebbe avere ad oggetto atti probatori. La "dipendenza" di cui parla l'art. 185 c.p.p. è infatti una dipendenza di tipo giuridico, predicabile tra due atti quando per legge - uno costituisca il presupposto necessario per il compimento dell'altro. È quanto accade per un atto di impulso processuale (come la citazione a giudizio) e gli atti del giudizio conseguente, che non può mai verificarsi per le prove, che non sono legate da alcuna connessione

51 CASSIBBA, Fabio, Brevi riflessioni sull'inutilizzabilità, cit., p. 11. In questa impostazione, affinché i materiali probatori reperiti grazie alla tortura siano utilizzabili, gli organi inquirenti dovrebbero «dimostrare che il loro reperimento non è in alcun modo il frutto della violazione del divieto di tortura e della coartata collaborazione processuale dell'imputato».

52 NOBILI, Massimo, La nuova procedura penale. Bologna: Clueb, 1989, p. 157 s.; COMOGLIO, Luigi Paolo, Perquisizione illegittima ed inutilizzabilità derivata delle prove acquisite con susseguente sequestro. Cassazione penale, Milano, n. 5 , p. 1547, 1996. 
giuridicamente necessaria, né tra loro, né rispetto alle decisioni che su di esse si fondino ${ }^{53}$.

Il caso paradigmatico è quello della perquisizione illegittima che conduca ad un sequestro ${ }^{54}$ : secondo la teoria cd. del male captum, bene retentum il vizio della perquisizione non inficerebbe il sequestro compiuto secondo le regole; diversamente, secondo la opposta poisonous tree's fruit doctrine, non sarebbe consentita l'acquisizione di quanto rinvenuto a seguito di una ricerca condotta in spregio delle previsioni che la disciplinano.

A quest'ultima conclusione può giungersi, evidentemente, solo a patto di concepire come un nesso di natura causale quello che consente la propagazione del vizio da una prova all'altra. Il fondamento normativo è rinvenuto nell'art. 191 c.p.p., leggendo l'espressione "prove acquisite" nel significato di "prove ottenute, reperite o raccolte" violando divieti legali: sarebbero perciò inutilizzabili tutte le prove che derivino, sulla base di un rapporto causale, da una violazione; non solo le prove vietate, ma «più in generale tutte quelle che siano l'esito di una violazione della legge» ${ }^{55}$.

53 CORDERO, Franco, Tre studi sulle prove penali, Milano: Giuffrè, 1963, p. 171, secondo il quale la valutazione in una decisione processuale di una prova invalida - nulla o inutilizzabile, o inesistente, come sarebbe una dichiarazione estorta tramite tortura - si risolve in ogni caso in un vizio della motivazione della decisione stessa. In senso conforme, SCELLA, Andrea, Prove penali e inutilizzabilità. Uno studio introduttivo, Torino: Giappichelli, 2000, p. 199; FERRUA, Paolo, Perquisizioni illegittime e sequestro: una singolare decisione di inammissibilità con effetti dissuasivi. Giurisprudenza costituzionale, n. 5, 2019, p. 2589.

Sulla questione si sono pronunciate dapprima le Sezioni unite, con una pronuncia contraddittoria, che negava in concreto ma teoricamente ammetteva la configurabilità di un simile meccanismo di propagazione (Cass., sez. un., 27 marzo 1996, Sala, in Diritto penale e processo, Milano, 1996, p. 1125), e poi la Corte costituzionale, che ha invece escluso l'applicabilità dell'art. 182 c.p.p. alla invalidità che si manifesti come inutilizzabilità (C. cost., 27 settembre 2001, n. 332, in Giurisprudenza costituzionale, Milano, n. 5, p. 2821, 2001) ed ha successivamente ribadito che un meccanismo di derivazione della inutilizzabilità, stante il principio di tassatività, avrebbe bisogno di una previsione normativa espressa (C. cost., 3 ottobre 2019, n. 219 in Giurisprudenza costituzionale, Milano, n. 5, p. 2589, 2019, con nota di FERRUA, Paolo, Perquisizioni illegittime e sequestro, cit.

In una variante di questa impostazione, il vizio della inutilizzabilità di una prova verrebbe "trasmesso" solo a quegli atti istruttori il cui legittimo 
A parte la seria controindicazione dell'estrema difficoltà che è possibile incontrare nella verifica di un nesso causale tra due o più prove, questa impostazione è priva di riscontro normativo: il verbo «acquisire» nell'art. 191 c.p.p. ha una caratura tecnica ineludibile, e un effetto di invalidazione a catena, come quello ipotizzato dai fautori della tesi dell'albero avvelenato, avrebbe richiesto ben altra e più precisa espressione.

Dichiarazioni o informazioni ottenute nei modi descritti dall'art. 613-bis c.p. - da considerarsi giuridicamente inesistenti - non possono essere adoperate in alcuna decisione (anche) istruttoria, del giudice o del p.m., per la quale il codice richieda una espressa giustificazione. Ma è solo in questo senso che possiamo assistere ad una sorta di "propagazione" della inutilizzabilità delle informazioni estorte nel procedimento penale.

In ogni caso, nessuna norma del nostro codice - neppure il comma 2-bis interpolato nell'art. 191 - può impedire che le dichiarazioni o le informazioni in discorso vengano "usate" come strumento puramente euristico. Il nesso tra una conoscenza rilevante (e magari processualmente non spendibile) e i passi investigativi successivi orientati in base ad essa è storico o psicologico, ma non giuridico.

È stato affermato che, per un'efficace "sterilizzazione" delle informazioni ottenute attraverso tortura, il legislatore avrebbe dovuto prescrivere la distruzione della documentazione, sulla falsariga di quanto previsto per le intercettazioni illegali. Tuttavia, misure simili, solitamente previste a tutela degli interessi extraprocessuali violati, sono poco adatte quale espediente per evitare che le indagini si avvantaggino di quelle informazioni o che il giudice ne sia condizionato psicologicamente: a parte che la distruzione presuppone qui l'accertamento definitivo del reato di tortura, il punto è che misure del genere rischiano non di eliminare ma di occultare, di rendere inverificabili i profitti investigativi delle pratiche illecite.

L'enfasi della formulazione non deve neppure indurre a ritenere che la mera presenza delle dichiarazioni estorte attraverso la tortura nello scacchiere istruttorio sia in grado di inquinare il giudizio e

compimento dipende dalla ricorrenza di determinati presupposti di fatto, come intercettazioni o perquisizioni (CAMON, Alberto, Le prove, in CAMON, Alberto; CESARI, Claudia; DANIELE, Marcello; DI BITONTO, Maria Lucia; NEGRI, Daniele; PAULESU, Pierpaolo, Fondamenti di procedura penale, Milano: Cedam, 2019, p. 319). 
conseguentemente la decisione: come si è anticipato, non diversamente da tutte le altre ipotesi in cui si voglia far valere l'uso decisorio di una informazione inutilizzabile, occorre sottoporre la decisione al consueto test di resistenza. In senso contrario, non è mancato chi ha letto la nuova norma nel senso che «ogni decisione giudiziaria e, segnatamente, la sentenza di condanna nei confronti della vittima di torture che tengano, comunque, conto delle dichiarazioni estorte sono affette, integralmente, da un'invalidità irrimediabile, che non può essere aggirata in sede d'impugnazione, attraverso il "test di resistenza" ${ }^{56}$. Si tratta, tuttavia, di una tesi difficilmente sostenibile: la dichiarazione estorta è probatoriamente inesistente, ma questo non significa che essa invalidi ex se la decisione; piuttosto, la sentenza che tragga fondamento dalla dichiarazione è affetta, in questa misura, da un vizio di motivazione, ma essa può essere confermata in appello o in sede di revisione sulla base di altre prove legittimamente acquisite e del tutto irrelate a quella ottenuta tramite tortura ${ }^{57}$.

Semmai il problema - relativo ad ogni prova invalida, sia nulla che inutilizzabile - è se il test di resistenza possa farlo anche la Corte di cassazione, come afferma la Suprema Corte, o se invece in quel caso il giudice di legittimità in realtà pretenda, sbagliando, di sostituirsi al giudice di merito, ripercorrendone il convincimento ${ }^{58}$.

Alla base della ricostruzione teorica ora accennata potremmo rinvenire considerazioni, non irragionevoli, attinenti segnatamente all'incidenza della tortura sull'affidabilità delle informazioni consegnate al processo: certe pratiche sono capaci di generare contesti di intimidazione in grado di inquinare anche attività investigative o probatorie diverse ed ulteriori rispetto a quella in seno alla quale sono state poste in essere; e tuttavia, l'antidoto a questo possibile effetto perturbante non può che essere una valutazione giudiziale particolarmente prudente ed attenta delle prove che possono esservi state esposte.

56

57

58

CASSIBBA, Fabio, Brevi riflessioni sull'inutilizzabilità, p. 114.

CORDERO, Franco, Procedura penale, cit.. p. 633; conforme FERRUA, Paolo, Prove illegittimamente acquisite, cit., p. 1255.

In argomento, cfr. CAPONE, Arturo, Il principio di decisività dei vizi della sentenza nel controllo della corte di cassazione, in Cassazione penale, Milano, 2004, p. 1463 ss. 
Sullo sfondo, c'è la comprensibile preoccupazione di interpretare la normativa nazionale in modo da prevenire condanne a Strasburgo, ma in questo caso, al di là degli effettivi spazi interpretativi, pare che si tratti di una preoccupazione non motivata. É vero che la Corte europea ha affermato che si può ritenere iniquo un processo, per lesione degli artt. 3 e 6 comma 1 C.e.d.u., in ragione del fatto che sia stata impiegata una dichiarazione estorta con tortura, ancorchè la stessa non costituisca prova decisiva ai fini della condanna ${ }^{59}$; ed è vero che i giudici di Strasburgo - che di solito lasciano agli Stati un ampio margine di apprezzamento nel costruire regole di esclusione o di valutazione della prova - postulano qui una sorta di divieto istruttorio (a differenza degli altri casi in cui, in presenza di attività probatoria lesiva di altri diritti protetti dalla Convenzione, segue il test della sole or decisive evidence rule). La violazione del divieto di tortura di cui all'art. 3 C.e.d.u. fa parte, infatti, del "nocciolo duro" delle tutele apprestate dalla Convenzione, inderogabile anche in caso di emergenza, e in linea di principio non "bilanciabile".

E tuttavia, secondo la giurisprudenza europea l'inosservanza dell'art. 3 C.e.d.u. è una condizione sì necessaria ma non sufficiente $\mathrm{a}$ rendere iniquo il processo ${ }^{60}$. Pur essendo improbabile che la Corte escluda una violazione dell'art. 6 C.e.d.u in presenza di pratiche di tortura, nel

59 C. eur. dir. uomo, sez. I, 30 aprile 2015, Shamardakof c. Russia, § 153 che «réitère sa jurisprudence selon laquelle l'utilisation d'éléments de preuve recueillis par la violation de l'un des droits absolus constituant le noyau dur de la Convention jette toujours de graves doutes quant à l'équité de la procédure, même si le fait d'avoir admis ces éléments comme preuves n'a pas été décisif pour la condamnation du suspect» (ribadisce la propria giurisperudenza secondo la quale l'uso di prove ottenute dalla violazione di uno dei diritti assoluti costituenti lo zoccolo duro della Convenzione solleva sempre seri dubbi - corsivo nostro - sull'equità del procedimento, anche se il fatto di aver ammesso questi elementi come prova non fosse decisivo per la condanna del sospettato).

60 Cfr. C. eur., G. C. 11 novembre 2006, Jalloh c. Germania, § 99 in Cassazione Penale, Milano, 2006, p 3843: «an issue may arise under Article $6 \S 1$ in respect of evidence obtained in violation of Article 3 of the Convention, even if the admission of such evidence was not decisive in securing the conviction» (un problema può - corsivo nostro - sorgere ai sensi dell'articolo $6 \S 1$ riguardo alle prove ottenute in violazione dell'articolo 3 della Convenzione, anche se l'ammissione di tali prove è stata non decisivo per ottenere la condanna). Peraltro nel caso di specie la corte aveva ritenuto decisiva la prova estorta. 
case law di Strasburgo non mancano precedenti in questo senso ${ }^{61}$, anche della Grande Camera ${ }^{62}$. Sembra perciò lecito affermare che l'impiego di prove ottenute attraverso tortura rappresenti un «grave indice di iniquità della procedura ${ }^{63}$, ma non una condizione automatica della stessa.

In questo senso, se il giudice d'impugnazione - rilevata l'inutilizzabilità - confermasse la condanna motivando su altre prove, residuerebbero al più spazi per ritenere violato l'art. 3 , ma non anche - di riflesso ed automaticamente - l'art. 6 C.e.d.u..

Viceversa, escludere la praticabilità della prova di resistenza per qualsiasi sentenza nella quale compaia in motivazione una dichiarazione ottenuta con la tortura significherebbe non solo introdurre una categoria di prove “anti-convenzionali” di cui non c'è traccia nella normativa ordinaria, ma rassegnarsi ad una sorta di effetto immunizzante della legge penale: commessa la tortura, il responsabile del reato per accertare il quale si è ricorsi alle sevizie non sarebbe più in alcun modo individuabile ${ }^{64}$.

Un autentico significato innovativo del comma 2-bis può essere rinvenuto, piuttosto, nella esclusione della possibilità che da quelle pratiche possano derivare informazioni suscettibili di costituire valide notitiae

61 C. eur., sez. I, 17 gennaio 2012, Alchagin c. Russia, § 73: «having regard to the foregoing, and, in particular, the applicant's own confession during the trial itself, the Court is unable to conclude, in the circumstances of this particular case, that the use of the applicant's confession statement made at the pre-trial stage rendered the proceedings against him wholly unfair» (tenuto conto di quanto precede e, in particolare, della confessione del ricorrente durante il processo stesso, la Corte non è in grado di concludere, nelle circostanze di questo caso particolare, che l'uso della dichiarazione di confessione del ricorrente resa nella fase istruttoria ha reso il procedimento contro di lui del tutto ingiusto). Nel caso già citato Camera Gäfgen c. Germania, §§ 10-46.

SPAGNOLO, Paola, Il modello europeo delle garanzie minime e il regime delle invalidità: un binomio conciliabile?, in MARANDOLA, Antonella, Le invalidità processuali, cit., p. 56. Peraltro, le conclusioni variano generalmente e si allineano al criterio della sole or decisive evidence quando le pratiche lesive dell'art. 3 c.e.d.u. siano configurabili non come tortura bensì come trattamento inumano e degradante, (C. eur. dir. uomo, 11 luglio 2006, Jalloh c. Germania, § 106107): il confine tra le due figure è però alquanto sfumato, evidentemente.

E' appena il caso di notare che la resistenza della motivazione della condanna dovrà essere saggiata eliminando, oltre alla prova derivante dalla tortura anche le prove che siano frutto di atti istruttori invalidi, in quanto motivati sulla base delle informazioni desunte da quella dichiarazione. 
criminis, in grado di dare inizio a nuovi procedimenti penali, a carico della stessa persona che quelle pratiche abbia subito o di terzi. Una "generica" comminatoria di inutilizzabilità probatoria, come quella contenuta nel primo comma dell'art. 191 c.p.p., non varrebbe ad escludere con sicurezza questa possibilità: l'atto dell'iscrizione di una notizia nell'apposito registro non ha i tratti di una 'decisione': non c'è alcuna utilizzazione probatoria dell'informazione, perché manca una delibazione di fondatezza; se pure volessimo intravedervi una qualche delibazione, saremmo comunque al di fuori del procedimento ${ }^{65}$.

Nella preclusione all'impiego delle informazioni in questione quale notizia di reato può insomma ravvisarsi il significato effettivamente precettivo di quella formula sanzionatoria "rafforzata" secondo cui essi non sono «comunque utilizzabili»: un regime analogo a quello dei documenti contenenti dichiarazioni anonime che, a mente dell'art. 240 c.p.p. non possono essere «in alcun modo utilizzati» o delle denunce anonime delle quali l'art. 333 c.p.p. afferma che «non può esser fatto alcun uso» ${ }^{66}$.

\section{Considerazioni conclusive.}

La novella del 2017, dunque, è un segnale forte, soprattutto a fronte di una recrudescenza del fenomeno delle "morti di Stato". Difficile, tuttavia, concludere l'excursus con un giudizio positivo sulla introduzione della nuova fattispecie. Al forte significato simbolico della novella, infatti, non sembra corrispondere un apparato di tutele che protegga la persona - imputato o testimone - da aggressioni tanto gravi e

65 Per questi rilievi, GABRIELLI, Chiara, Captazioni illecite come notizia di reato: dai ripensamenti del legislatore alle prime risposte della giurisprudenza., Cassazione penale, 2008, p. 1312.

66 Peraltro, da una denuncia anonima gli inquirenti possono trarre spunto per svolgere attività investigative dirette ad acquisire, nelle forme prescritte dal codice di rito, una notizia di reato (cfr. APRATI, Roberta, La notizia di reato nella dinamica del procedimento penale, Napoli: Jovene editore, 2010, p. 55 e, più di recente, NOCERINO, Wanda, Le denunce anonime come strumento di indagine. Un difficile equilibrio tra efficienza e garanzie. Diritto penale e processo, Milano, n. 12, p. 1607, 2017): la conclusione vale anche in relazione ad informazioni estorte con la tortura che non valgono ai fini dell'iscrizione, come detto, ma consentono di dare avvio all'attività di ricerca di una notitia criminis. 
l'accertamento processuale da possibili infiltrazioni di materiale acquisito attraverso condotte di tal fatta.

La novella sembra avere, più che altro, l'obiettivo - solo parzialmente raggiunto - di rassicurare l'interlocutore sovranazionale.

Analizzando l'art. 191, comma 2-bis, c.p.p., in effetti, si nota subito come la scelta di discostarsi in più punti dalla trama normativa di trattati e convenzioni nella redazione dell'incriminazione riverberi conseguenze negative anche sul versante processuale. La complessa formula contenuta nell'art. 613-bis c.p. rende complicato in tale dimensione l'accertamento del presupposto dal quale scaturisce la peculiare ipotesi di inutilizzabilità in questione. Essa inoltre si inserisce in un contesto nel quale operano già dispositivi collaudati, come quelli compendiati negli artt. 188 e 64 c.p.p., senza apportare vantaggi aggiuntivi significativi.

La disciplina rischia di compromettere anche il potenziale di deterrenza della previsione normativa. La sanzione di inutilizzabilità, infatti, non appare accompagnata da altre previsioni di supporto che ne regolino la applicazione e le sue conseguenze: come detto, la mancanza di una disciplina che chiarisca le modalità attraverso le quali, in assenza di un giudicato di condanna, accertare il compimento di atti di tortura affida all'interprete la gestione di uno snodo strategico senza, tuttavia, dotarlo degli strumenti processuali necessari. Parimenti opinabile appare la scelta di non regolare espressamente l'incidenza di una condotta rilevante ai sensi dell'art. 613-bis c.p. ai fini del giudizio di revisione.

\section{Biblografia}

ALESCI, Teresa, Il corpo umano fonte di prova. Padova: Cedam, 2017;

ALESSI, Giorgia, Il processo penale. Profilo storico. Roma - Bari: Laterza, 2001;

APRATI, Roberta, La notizia di reato nella dinamica del procedimento penale. Napoli: Jovene editore, 2010;

BECCARIA, Cesare, Dei delitti e delle pene. Milano: Mondadori, 1991;

BELTRANI, Sergio, Intercettazioni inutilizzabili e procedimento di prevenzione: la fine di un equivoco. Cassazione penale, 2010, p. 3063.

BRONZO, Pasquale, Accordi sul fascicolo e vizi degli atti. Cassazione penale, Milano, n. 11, p. 3764, 2020; 
BRONZO, Pasquale, Le modificazioni in tema di informazioni confidenziali, in LATTANZI, Giorgio (a cura di) Guida al giusto processo. Milano: Giuffrè, 2002;

CAMON, Alberto, Le prove, in A.A. VV., Fondamenti di procedura penale, Milano: Cedam, 2019;

CAPONE, Arturo, Il principio di decisività dei vizi della sentenza nel controllo della corte di cassazione. Cassazione penale, Milano, n. 4, p. 1463, 2004;

CAROLEO GRIMALDI, Nunzio, osservazioni a Cass., sez. V, 8 luglio 2019, n. 47079, in Cassazione penale, Milano, n. 6, p. 2349, 2020;

CASSIBBA, Fabio, Brevi riflessioni sull'inutilizzabilità delle dichiarazioni estorte con tortura ai sensi del nuovo art. 191, comma 2-bis, c.p.p., Diritto penale contemporaneo, Milano, n. 4, p. 109, 2018;

COLAIACOVO, Guido, L'imputato, in SPANGHER, Giorgio; MARANDOLA, Antonella; GARUTI, Giulio; KALB, Luigi (a cura di), Procedura penale. Teoria e pratica del processo. Torino: Utet, 2015;

COLELLA, Angela, C'è un giudice a Strasburgo. In margine alle sentenze sui fatti della Diaz e di Bolzaneto: l'inadeguatezza del quadro normativo italiano in tema di repressione penale della tortura. Rivista italiana di diritto e procedura penale, Milano, n. 4, p. 1801;

COLELLA, Angela, La repressione penale della tortura: riflessioni de iure condendo. Disponibile in https://archiviodpc.dirittopenaleuomo.org/upload/1406048334COLELLA_2014a.pdf;

COMOGLIO, Luigi Paolo, Perquisizione illegittima ed inutilizzabilità derivata delle prove acquisite con susseguente sequestro. Cassazione penale, Milano, n. 5, p. 1547, 1996;

CONTI, Carlotta, L'inutilizzabilità, in MARANDOLA, Antonella, Le invalidità processuali. Profili statici e dinamici. Torino: Utet, 2015;

CORDERO, Franco, Il procedimento probatorio, in Tre studi sulle prove penali. Milano: Giuffrè, 1963;

CORDERO, Franco, Procedura penale, IX ed. Milano: Giuffrè, 2012;

FERRUA, Paolo, Perquisizioni illegittime e sequestro: una singolare decisione di inammissibilità con effetti dissuasivi. Giurisprudenza costituzionale, Milano, n. 5, p. 2589, 2019;

FERRUA, Paolo, Prove illegittimamente acquisite: passato ed avvenire di un'illustre teoria. Diritto penale e processo, Milano, n. 9, p. 1249, 2020;

FOSCHINI, Gaetano, Sistema del diritto processuale penale, II ed. Milano: Giuffrè, 1965 ; 
GABRIELLI, Chiara, Captazioni illecite come notizia di reato: dai ripensamenti del legislatore alle prime risposte della giurisprudenza. Cassazione penale, n. 4 , p. 1312, 2008;

GALANTINI, Novella, L'inutilizzabilità della prova nel processo penale, Padova: Cedam, 1992;

GREVI, Vittorio, Nemo tenetur se detegere. Interrogatorio dell'imputato e diritto al silenzio nel processo penale italiano. Milano: Giuffrè, 1972;

GREVI, Vittorio; ILLUMINATI, Giulio, Prove, in CONSO, Giovanni; GREVI, Vittorio; BARGIS, Marta (a cura di), Compendio di procedura penale, IX ed. Padova: Cedam, 2018;

LOZZI, Gilberto, Lezioni di procedura penale. Torino: Giappichelli, 2018;

LUPARIA, Luca, La confessione dell'imputato nel sistema processuale penale. Milano: Giuffrè, 2006;

MAFFEI Stefano, Il mantello della legge. Male captum, bene retentum e dottrina del «ritrovamento inevitabile» in una recente pronuncia della Corte Europea dei diritti dell'uomo, in Studi in onore di Mario Pisani. Milano: Giuffrè, 2010, vol. 2;

MANZINI, Vincenzo, Trattato di diritto processuale penale italiano, Torino: Utet, 1931;

MAZZA, Oliviero, L'interrogatorio e l'esame dell'imputato nel suo procedimento. Milano: Giuffrè, 2004;

NEGRI, Daniele, Compressione dei diritti di libertà e principio di proporzionalità, in AA.VV., Diritti della persona e nuove sfide del processo penale, Milano: Giuffrè Francis Lefebvre, 2019;

NEGRI, Stefania, "Violazioni strutturali” e ritardo nell'esecuzione delle sentenze CEDU: il caso Cestaro $c$. Italia e l'incerta introduzione del reato di tortura nel codice penale italiano. Diritto penale e processo, Milano, n. 12, p. 1659, 2016;

NOBILI, Massimo, La nuova procedura penale. Bologna: Clueb, 1989;

NOCERINO, Wanda, Le denunce anonime come strumento di indagine. Un difficile equilibrio tra efficienza e garanzie. Diritto penale e processo, Milano, n. 12, p. 1607, 2017.

PELISSERO, Marco, L'introduzione del delitto di tortura nell'ordinamento italiano, in GIARDA, Angelo; GIUNTA, Fausto; VARRASO, Gianluca (a cura di), Dai decreti attuativi della legge "Orlando" alle novelle di fine legislatura. Padova: Cedam, 2018; PEZZIMENTI, Carmela, Tortura e diritto penale simbolico: un binomio indissolubile. Diritto penale e processo, Milano, n. 2, p. 153, 2018; 
ROMANELLI, Bartolomeo, Delitto di tortura e inutilizzabilità probatoria, in GIARDA, Angelo; GIUNTA, Fausto;

ROSSO NELSON, Rocco Antonio Rangel, La prova illecita nell'ordinamento processuale penale brasiliano, https://archiviodpc.dirittopenaleuomo.org/upload/5017-nelson2018a.pdf.

SCELLA, Andrea, Prove penali e inutilizzabilità. Uno studio introduttivo, Torino: Giappichelli, 2000;

VARRASO, Gianluca (a cura di), Dai decreti attuativi della legge "Orlando" alle novelle di fine legislatura. Padova: Cedam, 2018;

SPAGNOLO, Paola, Il modello europeo delle garanzie minime e il regime delle invalidità: un binomio conciliabile?, in MARANDOLA, Antonella, Le invalidità processuali. Profili statici e dinamici. Torino: Utet, 2015;

SPAGNOLO, Paola, L'ordine europeo di indagine penale, in MARCHETTI, Maria Riccarda; SELVAGGI, Eugenio (a cura di), La nuova cooperazione giudiziaria penale. Padova: Cedam, 2019;

UBERTIS, Giulio, Sistema di procedura penale, vol. II. Milano: Giuffrè, 2020.

\section{Additional information and author's declarations (scientific integrity)}

Conflict of interest declaration: the authors confirm that there are no conflicts of interest in conducting this research and writing this article.

Declaration of authorship: all and only researchers who comply the authorship requirements of this article are listed as authors; all coauthors are fully responsible for this work in its entirety. Lo scritto è frutto di riflessioni comuni degli autori. La redazione dei paragrafi da 1-4 e 6 è stata curata da Guido Colaiacovo, quella del paragrafo 5 da Pasquale Bronzo.

- Pasquale Bronzo: conceptualization, methodology, data curation, investigation, writing - original draft, validation, writing - review and editing, final version approval. 
- Guido Colaiacovo: conceptualization, methodology, data curation, investigation, writing - original draft, validation, writing - review and editing, final version approval.

Declaration of originality: the authors assure that the text here published has not been previously published in any other resource and that future republication will only take place with the express indication of the reference of this original publication; they also attest that there is no third party plagiarism or self-plagiarism.

\section{Editorial process information}

(http://www.ibraspp.com.br/revista/index.php/RBDPP/about/editorialPolicies)

- Submission date: 30/12/2020

- Preliminary control and similarity verification: 06/01/2021

- Review 1: 31/01/2021

- Review 2: 31/01/2021

- Editorial decision 1: 04/02/2021

- Correction round return: 02/03/2021

- Final editorial decision: 03/03/2021
Editorial team responsible

- Editor-in-chief: 1 (VGV)

- Associated-editor: 2 (KK e BC)

- Reviewers: 2

\section{HOW TO CITE (ABNT BRAZIL):}

BRONZO, Pasquale; COLAIACOVO, Guido. Inutilizzabilità delle prove e delitto di tortura nel sistema processuale italiano. Revista Brasileira de Direito Processual Penal, Porto Alegre, vol. 7, n. 1, p. 311-342, jan./abr. 2021. https://doi.org/10.22197/rbdpp.v7i1.508

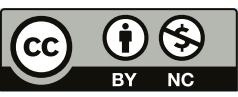

Esta obra está licenciada com uma Licença Creative Commons Atribuição-NãoComercial 4.0 Internacional. 